\title{
Electron Detachment in Negative-Ion Collisions. II. The Dynamical Complex Potential
}

\section{T. S. Wang}

John B. Delos

William \& Mary, jbdelos@wm.edu

Follow this and additional works at: https://scholarworks.wm.edu/aspubs

Part of the Physics Commons

\section{Recommended Citation}

Early experiments on collisional detachment of electrons from negative ions were interpreted in terms of a "local" or "static" complex potential. In the preceding paper we developed a more general close-coupling framework for describing such collisions. In this theory, the amplitude for finding the electron in the bound state $\mathrm{C}-1(\mathrm{t})$ satisfies an integro-differential equation. We define an "exact" or "dynamical" complex potential $\mathrm{E}(\mathrm{t})$ such that $\mathrm{C}-1(\mathrm{t}) \equiv \exp \left[-\mathrm{i} \int \mathrm{t} 0 \mathrm{t} E\left(\mathrm{t}^{\prime}\right) \mathrm{dt} \mathrm{t}^{\prime} \hbar\right]$. An integral equation for $\mathrm{E}(\mathrm{t})$ is derived and solved. It is found that in slow collisions, the dynamical complex potential oscillates about the static complex potential. Approximate formulas for $\mathrm{E}(\mathrm{t})$ are given.

This Article is brought to you for free and open access by the Arts and Sciences at W\&M ScholarWorks. It has been accepted for inclusion in Arts \& Sciences Articles by an authorized administrator of W\&M ScholarWorks. For more information, please contact scholarworks@wm.edu. 


\title{
Electron detachment in negative-ion collisions. II. The dynamical complex potential
}

\author{
T. S. Wang and J. B. Delos \\ Physics Department, The College of William and Mary, Williamsburg, Virginia 23185
}

(Received 23 June 1983)

\begin{abstract}
Early experiments on collisional detachment of electrons from negative ions were interpreted in terms of a "local" or "static" complex potential. In the preceding paper we developed a more general close-coupling framework for describing such collisions. In this theory, the amplitude for finding the electron in the bound state $C_{-1}(t)$ satisfies an integro-differential equation. We define an "exact" or "dynamical" complex potential $\mathscr{E}(t)$ such that $C_{-1}(t) \equiv \exp \left[-i \int_{t_{0}}^{t} \mathscr{E}\left(t^{\prime}\right) d t^{\prime} / \hbar\right)$. An integral equation for $\mathscr{E}(t)$ is derived and solved. It is found that in slow collisions, the dynamical complex potential oscillates about the static complex potential. Approximate formulas for $\mathscr{E}(t)$ are given.
\end{abstract}

\section{INTRODUCTION}

In the preceding paper, ${ }^{1}$ it was shown that processes involving transitions from a discrete state to a continuum can be described in the semiclassical close-coupling framework by a set of coupled differential equations, and that under assumptions believed to be quite general, the solution to those equations can be obtained from the solution to a single integro-differential equation

$$
i \hbar \frac{d}{d t} C_{-1}(t)=\Delta(t) C_{-1}(t)+\int_{t_{0}}^{t} \mathscr{G}\left(t, t^{\prime}\right) C_{-1}\left(t^{\prime}\right) d t^{\prime},
$$

where $C_{-1}(t)$ is the amplitude for finding the system in the bound state as a function of time, i.e., it is the probability amplitude for survival of the negative ion. The purpose of this paper is to find solutions to this fundamental equation (1.1).

For the rest of this paper, we will be considering only $C_{-1}(t)$ and not $C_{\epsilon}(t)$, so we will simplify the notation by dropping the subscript, writing

$$
C_{-1}(t) \equiv C(t) \text {. }
$$

In early treatments of electron detachment ${ }^{2-4}$ it was assumed that the unstable negative molecular ion $A B^{-}$, which is temporarily formed in the collision, decays by electron emission according to an exponential law, so that the survival probability is given by

$$
P_{s}(t)=|C(t)|^{2}=\exp \left[-\int_{t_{0}}^{t} \Gamma\left[R\left(t^{\prime}\right)\right] d t^{\prime} / \hbar\right] .
$$

$\frac{1}{2} \Gamma$ can be regarded as the imaginary part of a local complex potential. This assumption of exponential decay follows a long tradition in physics, one that goes back to the first studies of the decay of radioactive nuclei. ${ }^{5}$ In a study of atomic autoionization, Fano ${ }^{6}$ showed that if an isolated state is imbedded high in a continuum, then the decay is indeed exponential, and

$$
\Gamma=2 \pi\left|V_{-1, \epsilon}\right|^{2} \rho_{\epsilon},
$$

where $V_{-1, \epsilon}$ is the coupling matrix element between discrete and continuum states, and $\rho_{\epsilon}$ is the density of continuum states. Later Demkov and his collaborators ${ }^{7}$ showed that if a discrete state moves through a continuum linearly with time, and if the coupling matrix elements $V_{-1, \epsilon}(t)$ are independent of time, then the exponential decay law again holds. However, the assumptions made in these derivations cannot be valid for most negative-ion systems, because experiments have shown unambiguously that the exponential decay law with a local complex potential sometimes makes incorrect predictions about isotope effects. ${ }^{8-11}$

By solving the fundamental equation (1.1), we can find the exact behavior of the survival amplitude $C(t)$ [subject, of course, to the assumptions made in deriving Eq. (1.1)]; this enables us to find conditions under which exponential decay occurs, and it enables us to give an accurate description of systems that decay nonexponentially.

Let us define $\mathscr{E}(t)$ such that

$$
C(t)=\exp \left[-i \int_{t_{0}}^{t} \mathscr{E}\left(t^{\prime}\right) d t^{\prime} / \hbar\right],
$$

i.e.,

$$
\mathscr{C}(t)=i \hbar \dot{C}(t) / C(t)
$$

For all $t$ such that $C(t) \neq 0, \mathscr{E}(t)$ is finite, continuous, and differentiable. Substituting (1.6) into (1.1), we obtain an integral equation for $\mathscr{E}(t)$,

$$
\mathscr{E}(t)=\Delta(t)+\int_{t_{0}}^{t} \mathscr{G}\left(t, t^{\prime}\right) \exp \left[i \int_{t^{\prime}}^{t} \mathscr{E}\left(t^{\prime \prime}\right) d t^{\prime \prime} / \hbar\right] d t^{\prime},
$$

with the boundary condition

$$
\mathscr{E}\left(t_{0}\right)=\Delta\left(t_{0}\right) \text {. }
$$

We take $t_{0}$ to be a time long before the collision, when $\mathscr{G}\left(t, t^{\prime}\right)$ is insignificant. Then $\mathscr{E}(t)$ remains equal to $\Delta(t)$ until $\mathscr{G}\left(t, t^{\prime}\right)$ becomes substantial, at which time $t-t_{0}$ is large. Changing to variables $t, \tau=t-t^{\prime}$, Eq. (1.7) becomes 


$$
\begin{aligned}
\mathscr{E}(t)= & \Delta(t) \\
& +\int_{0}^{t-t_{0}} \mathscr{G}(\tau ; t) \exp \left[i \int_{t-\tau}^{t} \mathscr{E}\left(t^{\prime \prime}\right) d t^{\prime \prime} / \hbar\right) d \tau .
\end{aligned}
$$

The analysis above is exact, and (1.9) is just another form of the fundamental equation (1.1). However, $\mathscr{E}(t)$ is amenable to a physical interpretation: Because of the close relationship between (1.5) and (1.3), if the localcomplex-potential model were correct, then $\operatorname{Im}[\mathscr{E}(t)]$ would have to be equal to $\frac{1}{2} \Gamma(R(t))$. But since $\mathscr{E}(t)$ is defined by its relation to the exact solution to (1.1), we may think of it as an exact or dynamical complex potential, which may or may not be close to the local or static complex potential.

In this paper we shall give ways of calculating $\mathscr{E}(t)$ by solving (1.9), and we shall show that for slow collisions the dynamical complex potential approaches the static complex potential. However, we shall also show that they cannot be identical; they differ in some obvious ways and in some surprising ways.

\section{SLOW COLLISION APPROXIMATION AND THE LOCAL-COMPLEX-POTENTIAL FORMULAS}

In this section it is shown that for slow collisions, $\mathscr{E}(t)$ tends to approach the local complex potential. To establish this, we must assume that the time scale on which $\mathscr{E}(t)$ changes is comparable to that on which $\Delta(t)$ and $V_{-1, \epsilon}(t)$ change, and that this time scale is long compared to the time $\Delta \tau$ on which $\mathscr{G}(\tau ; t)$ is significant. Then in (1.9) we can replace $\mathscr{E}\left(t^{\prime \prime}\right)$ by $\mathscr{E}(t)$, to obtain

$$
\mathscr{E}(t)=\Delta(t)+\int_{0}^{t-t_{0}} \mathscr{G}(\tau ; t) \exp [i \mathscr{E}(t) \tau / \hbar] d \tau
$$

Since $\Delta \tau$ is also small compared to $t-t_{0}$, we can replace the upper limit by infinity. We then recognize that the integral in (2.1) is just the Fourier transform of $\mathscr{G}$ that was introduced in Eq. (3.17) of the preceding paper. ${ }^{1}$ Consistent with the approximation $\mathscr{E}\left(t^{\prime \prime}\right) \simeq \mathscr{E}(t)$, which was already made, we may also use the short-memory approximation, $V_{-1, \epsilon}\left(t^{\prime}\right) \simeq V_{-1, \epsilon}(t)$, which leads us to Eq. (3.19) of Ref. 1. Thus for slow collisions, $\mathscr{E}(t)$ is approximately equal to the solution to the equation

$$
\mathscr{E}(t)=\Delta(t)+G_{s}(\mathscr{E}(t) ; t)
$$

Let us define $\mathscr{C}_{s}(t)$ as the exact solution to this approximate equation (2.2). Once $G_{s}(\epsilon ; t)$ is known, $\mathscr{C}_{s}(t)$ can be obtained by an iterative algebraic process: The zero-order approximation is

$$
\mathscr{C}_{s}^{(0)}(t)=\Delta(t)
$$

and, substituting this back into (2.2), the first-order approximation is

$$
\mathscr{E}_{s}^{(1)}(t)=\Delta(t)+G_{s}(\Delta(t) ; t)
$$

and so on. For a slow collision, the exact $\mathscr{E}(t)$, defined in Eq. (1.5), will be close to $\mathscr{C}_{s}(t)$.

Equations (2.2) and (2.4) represent two different versions of the classical local-complex-potential formulas; if this is not already clear, we may use Eq. (3.20) of the preceding paper $^{1}$ to obtain

$$
\operatorname{Im}\left[\mathscr{E}_{s}^{(1)}(t)\right]=-\pi\left|V_{-1, \Delta(t)}(t)\right|^{2} \rho_{\Delta(t)},
$$

which corresponds directly to (1.4).

It is very pleasing to arrive in this way at the localcomplex-potential formulas because they are familiar results, known to be applicable to some systems. The above is one of the most general derivations of these formulas that has yet been given. Other treatments have relied upon the hypothesized existence of a long-lived quasibound resonance, and Taylor and Delos's treatment (following Demkov) made use of special assumptions about $\Delta(t)$ and $V_{-1, \epsilon}(t)$. The present derivation used only the general assumptions listed in the preceding paper and the approximations leading from (1.9) to (2.2).

On the other hand, it is well known that the classical local-complex-potential formulas have some problems: It is not that they are slightly inaccurate, but rather that they give a qualitatively incorrect description of some systems-for $\mathrm{H}^{-}\left(\mathrm{D}^{-}\right)$on $\mathrm{Ne}, \mathrm{Ar}, \mathrm{Kr}$, or $\mathrm{Xe}$, they predict an isotope effect that is opposite to what is observed. This is because they do not properly describe phenomena that arise when the discrete curve just grazes the continuum. A related but rather subtle inconsistency of the above equations also appears when calculations are made. We showed that $\hat{G}(\epsilon)$ has a discontinuous derivative at $\epsilon=0$; $G_{s}(\epsilon ; t)$ must have the same behavior. As a consequence, $\mathscr{E}_{s}(t)$, the solution to Eq. (2.2), will have a discontinuous derivative with respect to time when $\mathscr{E}_{s}=0$. However, in Appendix A of Ref. 1, we took some care to prove that $C_{-1}(t), d C_{-1} / d t$, and $d^{2} C_{-1} / d t^{2}$ all are continuous functions of time, and this implies that the exact $\mathscr{E}(t)$ has a continuous derivative. The exact or dynamical complex potential must go smoothly through the region where the static local complex potential has bumps. ${ }^{12}$

\section{ITERATIVE CALCULATION OF $\mathscr{E}(t)$ AND FIRST-ORDER APPROXIMATION}

Recognizing the limitations of the slow-collision approximation given in the preceding section, we present here an iterative method to find $\mathscr{E}(t)$ and we show the results of a "first-order" calculation. It is found that $\mathscr{C}^{(1)}(t)$, obtained from this first-order calculation, is comparable to that obtained in the local-complex-potential approximation, but this first-order approximation also describes tunneling and interference effects.

Returning to Eq. (1.9), the zero-order approximation to $\mathscr{E}(t)$ is of course

$$
\mathscr{C}^{(0)}(t)=\Delta(t),
$$

and the iterative scheme for solving (1.9) is obvious: given an " $n$ th-order" approximation $\mathscr{C}^{(n)}(t)$, the " $(n+1)$ thorder" approximation is 


$$
\mathscr{E}^{(n+1)}(t)=\Delta(t)+\int_{0}^{t-t_{0}} \mathscr{G}(\tau ; t) \exp \left[i \int_{t-\tau}^{t} \mathscr{E}^{(n)}\left(t^{\prime}\right) d t^{\prime} / \hbar\right] d \tau
$$

The "zeroth-order" approximation (3.1), together with Eqs. (2.13) and (3.3) of Ref. 1, lead to

$$
C_{\epsilon}(t)=(i \hbar)^{-1} \exp \left[-i \epsilon\left(t-t_{0}\right) / \hbar\right] \int_{t_{0}}^{t} d t^{\prime} V_{\epsilon,-1}\left(t^{\prime}\right) \exp \left[i \int_{t_{0}}^{t^{\prime}}\left[\epsilon-\Delta\left(t^{\prime \prime}\right)\right] d t^{\prime \prime} / \hbar\right]
$$

which is easily seen to be the result of first-order timedependent perturbation theory applied to the original coupled equations (2.8). ${ }^{1}$ This approximation was used recently to calculate cross sections for collisions of $\mathrm{H}^{-}$and $\mathrm{D}^{-}$with $\mathrm{Ne}^{13}$ On the other hand, although the zeroorder approximation coincides with first-order perturbation theory, there is in general no correspondence between the present $n$ th-order approximation and any level of the standard perturbation theory; even our first-order approximation given below contains parts of all orders of perturbation theory.

In the present paper, we will calculate only the firstorder approximation. Furthermore, to reduce the amount of uninteresting complexity, we use the separable approximation and the short-memory approximation for $\mathscr{G}(\tau ; t)$, so the propagator takes the form (3.23). ${ }^{1}$ For a simple but not unrealistic calculation, we may take

$$
\begin{aligned}
& g(t) \simeq 1 \\
& \Delta(t)=E_{0}-\beta t^{2}
\end{aligned}
$$

We then have

$$
\begin{array}{r}
\mathscr{E}^{(1)}(t)-\Delta(t)=\int_{0}^{\infty} d \tau \hat{\mathscr{G}}(\tau) \exp \left[i \int_{t-\tau}^{t} \Delta\left(t^{\prime}\right) d t^{\prime} / \hbar\right) \\
=\int_{0}^{\infty} \hat{\mathscr{G}}(\tau) \exp \left\{i \left[\left(E_{0}-\beta t^{2}\right) \tau\right.\right. \\
\left.\left.+\beta t \tau^{2}-\frac{1}{3} \beta \tau^{3}\right]\right\} d \tau
\end{array}
$$

[ $\hbar=1$ in (3.6) and below].

We have seen in Fig. 1 of Ref. 1 that $\hat{G}(\tau)$ gets small as $\tau$ gets large. So, temporarily neglecting the terms involving $\tau^{2}$ and $\tau^{3}$ in the exponent, we obtain

$$
\begin{aligned}
\mathscr{E}^{(1)}(t)-\Delta(t) & \simeq \int_{0}^{\infty} d \tau \hat{\mathscr{G}}(\tau) \exp \left[i\left(E_{0}-\beta t^{2}\right) \tau\right] \\
& =\widehat{G}\left(E_{0}-\beta t^{2}\right) .
\end{aligned}
$$

This is a special case of the slow-collision approximation that was discussed in the preceding section, and we have again arrived at the local-complex-potential formula.

The neglected terms in the exponent of Eq. (3.6) have surprising effects: they give oscillations in $\mathscr{C}(t)$. To see this, let us define

$$
\begin{aligned}
& f_{1}(\tau ; t)=\left(E_{0}-\beta t^{2}\right) \tau, \\
& f_{2}(\tau ; t)=f_{1}(\tau ; t)+\beta t \tau^{2}, \\
& f_{3}(\tau ; t)=f_{2}(\tau ; t)-(\beta / 3) \tau^{3},
\end{aligned}
$$

i.e., $f_{k}(\tau ; t)$ is a $k$ th degree approximation to the exponent in Eq. (3.6). We may also define

$$
F_{k}(t) \equiv \int_{0}^{\infty} \hat{\mathscr{G}}(\tau) \exp \left[i f_{k}(\tau ; t)\right] d \tau
$$

In Fig. 1 we show $F_{1}(t)$ and $F_{2}(t)$, and in Fig. 2 we show $F_{2}(t)$ and $F_{3}(t)$. These all are numerical evaluations of Eq. (3.10) for $E_{0}=0.2$ and $^{14} \beta=5 \times 10^{-6}$.

$F_{1}(t)$ is given in (3.8), and it is the local complex potential [minus $\Delta(t)$ ]. It is a symmetric function of $t$, and it has a discontinuous derivative at the times $\pm t^{x}$ $= \pm\left(E_{0} / \beta\right)^{1 / 2}$, when $\Delta(t)=0$. This is the time at which the discrete state crosses into or out of the continuum; there the real part of $\hat{G}$ has a cusp, and the imaginary part goes to zero as $\left|t-t^{x}\right|^{1 / 2}$. In Fig. 1 it is seen that $F_{2}(t)$ stays close to $F_{1}(t)$ for $0<t<t^{x}$, but that $F_{2}(t)$ oscillates about $F_{1}(t)$ for $t^{x} \leq t$. (In the present case $t_{\text {out }}^{x}=200$ a.u.)

In Fig. 2 we see that the oscillations in $F_{2}(t)$ also occur when $-t^{x}<t<0$ (these oscillations damp out for $\left.0<t<t^{x}\right)$, whereas $F_{3}(t)$ has some oscillations for all $t>-t^{x}$. Figure 3 compares $F_{1}$ (the local complex potential) and $F_{3}$, the first-order approximation to the dynamical complex potential.

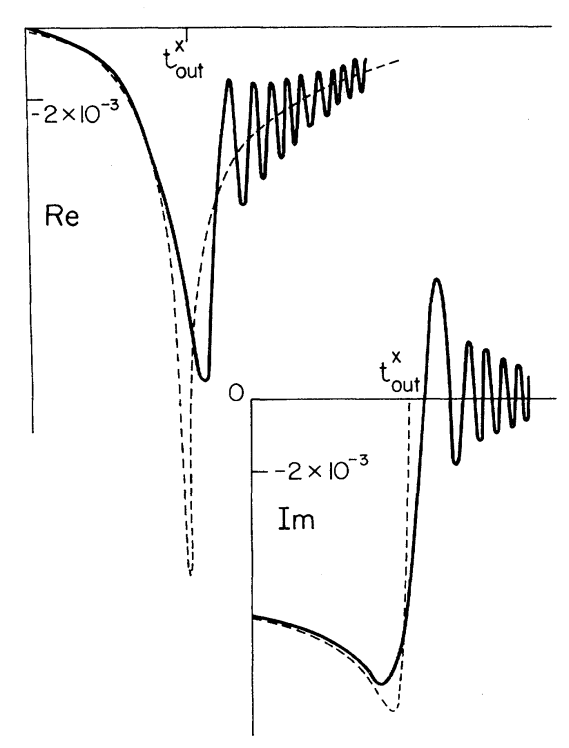

FIG. 1. $F_{1}(t)$ (dashed line) and $F_{2}(t)$ (solid line) for $t>0 . \quad F_{1}$ and $F_{2}$ are defined in Eqs. (3.9) and (3.10). $F_{1}(t)$ is the same as $\widehat{G}(\Delta(t))$, so it represents a part of the local complex potential. Its real part has a cusp at the point where the discrete curve crosses into the continuum, and its imaginary part goes to zero there. $\left[\Delta(t)+F_{2}(t)\right]$ is an approximation to the dynamical complex potential. It is continuous, and oscillates about the local complex potential. 

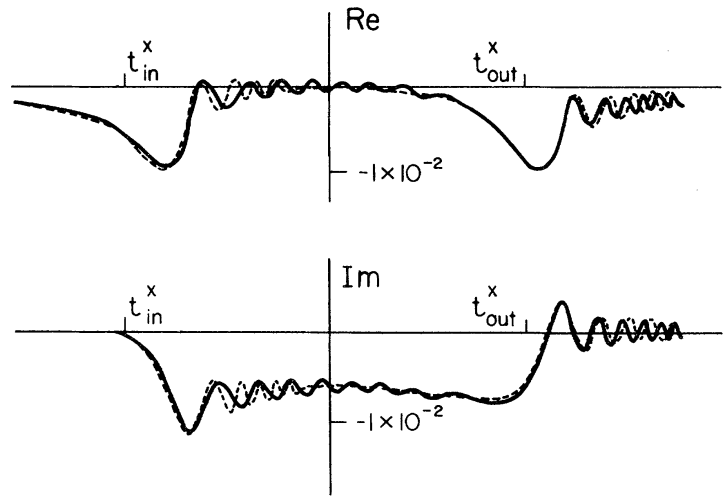

FIG. 2. $F_{2}(t)$ (dashed line) and $F_{3}(t)$ (solid line) defined in Eqs. (3.9) and (3.10) for all $t$. $\left[\Delta(t)+F_{3}(t)\right]$ is a better approximation to the dynamical complex potential. It is somewhat more oscillatory than $F_{2}(t)$.

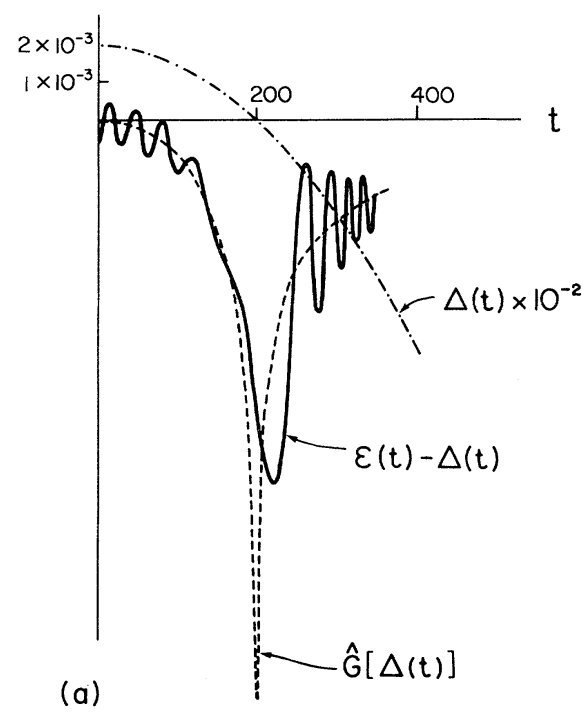

(b)

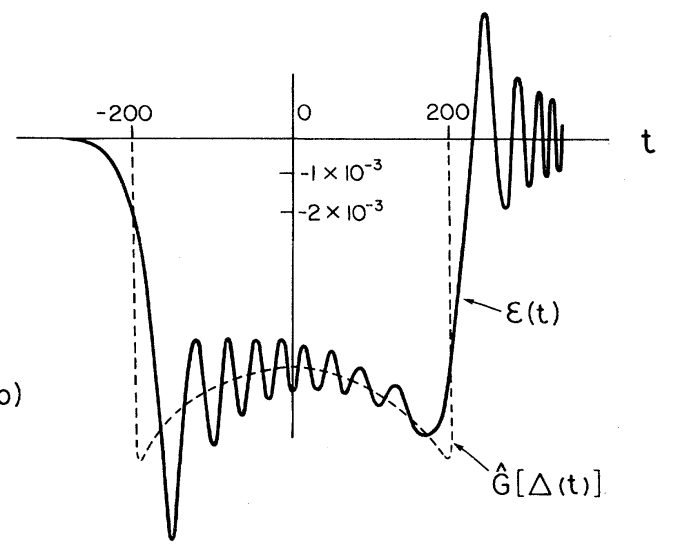

FIG. 3. (a) Comparison of static and dynamical complex potentials. Here we show the real part for $t>0$. Dotted-dashed line is the energy gap between the discrete state and the continuum, $\Delta(t) \times 10^{-2}$. Dashed line is $\operatorname{Re} \hat{G}(\Delta(t)) \equiv \operatorname{Re} F_{1}(t)$, part of the static or local complex potential. Solid line is $\operatorname{Re}[\mathscr{C}(t)-\Delta(t)] \equiv \operatorname{Re}\left(F_{3}(t)\right)$, the corresponding part of the dynamical complex potential. (b) As in (a), but here is the imaginary part.

\section{SIMPLIFYING APPROXIMATIONS}

We can better understand these results by considering some simplifying approximations. Contributions to the integral appearing in Eqs. (3.5) and (3.6) mainly come from certain small ranges of $\tau$. For general values of $\tau$, $\hat{\mathscr{Y}}(\tau)$ is small and the exponent is rapidly varying, so the contributions come from the region near $\tau=0$, where $\hat{\mathscr{Y}}(\tau)$ is large, and from stationary phase points, where the exponent is slowly varying.

The contribution from the region near $\tau=0$ was already estimated by using the slow-collision approximation

$$
\begin{aligned}
\int_{0}^{\tau_{\infty}} d \tau \hat{\mathscr{G}}(\tau) \exp \left[i \int_{t-\tau}^{t} \Delta\left(t^{\prime}\right) d t^{\prime} / \hbar\right] \\
\simeq \int_{0}^{\tau_{\infty}} \hat{\mathscr{Y}}(\tau) \exp [i \tau \Delta(t) / \hbar] d \tau=\hat{G}[\Delta(t)] .
\end{aligned}
$$

Here $\tau_{\infty}$ is a value of $\tau$ that is sufficiently large that $\hat{\mathscr{G}}\left(\tau_{\infty}\right)$ is small, but sufficiently small that $\Delta(t)$ does not change significantly in the range from $t-\tau$ to $t$.

Stationary phase contributions to the integral arise from regions near the points $\tau_{s p}$ at which

$$
\begin{aligned}
& \left.\qquad \frac{d}{d \tau} \int_{t-\tau}^{t} \Delta\left(t^{\prime}\right) d t^{\prime}\right]_{\tau_{s p}}=\Delta\left(t-\tau_{s p}\right)=0, \\
& \text { i.e., } \\
& \qquad \tau_{s p}=t-t^{x}
\end{aligned}
$$

where $t^{x}$ is a time at which $\Delta(t)=0$. With the quadratic approximation to $\Delta(t)$, there are two such points, $t_{\mathrm{in}}^{x}$ and $t_{\text {out }}^{x}$, where the discrete curve crosses into the continuum and where it crosses back out. However, the integral in Eq. (3.5) involves only positive values of $\tau$, so the stationary phase contributions should only be considered for $t_{\text {in }}^{x}<t$. Hence we have the following cases: $t<t_{\text {in }}^{x}$, no stationary phase contribution; $t_{\text {in }}^{x}<t<t_{\text {out }}^{x}$, one stationary phase contribution, near $t_{\text {in }}^{x} ; t_{\text {out }}^{x}<t$, two stationary phase contributions, near $t_{\mathrm{in}}^{x}$ and $t_{\mathrm{out}}^{x}$. In each case, the contribution to the integral from the region of stationary phase is

$$
\begin{aligned}
\left|\frac{2 \pi}{\dot{\Delta}\left(t_{n}^{x}\right)}\right|^{1 / 2} \exp \left[i(\pi / 4) \operatorname{sgn} \dot{\Delta}\left(t_{n}^{x}\right)\right] \widehat{\mathscr{G}}\left(t-t_{n}^{x}\right) \\
\quad \times \exp \left[i \int_{t-t_{n}^{x}}^{t} \Delta\left(t^{\prime}\right) d t^{\prime} / \hbar\right] .
\end{aligned}
$$

The integral in Eq. (3.6) is then estimated by combining (4.1) with zero, one, or two terms of the form (4.3). From the quadratic approximation, $\Delta(t)=E_{0}-\beta t^{2}$, with $E_{0}=0.2$ and $\beta=5 \times 10^{-6}$, the result is shown in Fig. 4 ; it is very close to the result obtained by numerical evaluation of Eq. (3.6).

Why do oscillations appear in $\mathscr{E}(t)$ and what do they mean? In discrete curve-crossing problems, Stueckelberg ${ }^{15}$ showed in 1932 that the transition probability would be 
oscillatory because of interference between transitions that occur on incoming and outgoing parts of a trajectory. Similar oscillations are also known to occur in Penning ionization, which involves transitions between a discrete state and a continuum. Such oscillations are also possible in systems undergoing electron detachment (though they have not yet been seen in experiments). Oscillations in the transition probability must also manifest themselves in $C(t)$, and, therefore, also in the dynamical complex potential $\mathscr{E}(t)$. Like Stueckelberg oscillations, the phase of the oscillatory part of Eq. (4.3)

$$
\int_{t-t^{x}}^{t} \Delta\left(t^{\prime}\right) d t^{\prime} / \hbar
$$

is related to the integral of the energy gap $\Delta(t)$ from the time of crossing to $t$.

\section{CONCLUSION}

We have shown that the survival amplitude $C(t)$ can be written in the form (1.5), and that $\mathscr{E}(t)$ then plays the role of a dynamical complex potential. It satisfies an integral equation (1.7) for which an approximate solution is

$$
\mathscr{E}^{(1)}(t)=\Delta(t)+\int_{0}^{t-t_{0}} \mathscr{G}(\tau ; t) \exp \left[i \int_{t-\tau}^{t} \Delta\left(t^{\prime}\right) d t^{\prime} / \hbar\right] d \tau,
$$

and further approximations to the integral give

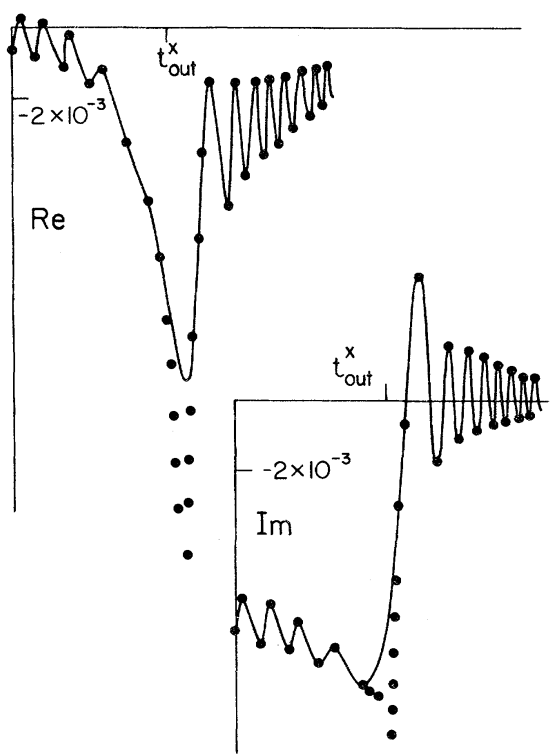

FIG. 4. Test of the approximation defined by Eqs. (4.1) and (4.3). Solid line: Dynamical complex potential $\mathscr{E}(t)-\Delta(t) \equiv F_{3}(t)$. Dotted line: Static complex potential $\widehat{G}(\Delta(t))$ plus contributions from stationary phase points (4.3). The approximation is evidently very accurate except in small regions where the discrete curve crosses into or out of the continuum.

$$
\mathscr{E}^{(1)}(t) \simeq G[\Delta(t) ; t]+\sum_{n}\left|\frac{2 \pi}{\dot{\Delta}\left(t_{n}^{x}\right)}\right|^{1 / 2} \exp \left[i\left[\int_{t-t_{n}^{x}}^{t} \Delta\left(t^{\prime}\right) d t^{\prime} / \hbar+(\pi / 4) \operatorname{sgn} \dot{\Delta}\left(t_{x}^{n}\right)\right]\right] \mathscr{G}\left(t-t_{n}^{x} ; t\right),
$$

where the sum is over times $t_{n}^{x}<t$ for which $\Delta\left(t_{n}^{x}\right)=0$. Numerical calculations show that the dynamical complex potential oscillates about the static complex potential. In a future paper (in preparation), we will use this method to calculate cross sections.

\section{ACKNOWLEDGMENTS}

Portions of this paper are based on the Ph.D. dissertation by one of us (T.S.W.), College of William and Mary, 1983. Support for this project was provided by the National Science Foundation and the Jeffress Foundation.
1T. S. Wang and J. B. Delos, preceding paper, Phys. Rev. A 29 , 542 (1984).

${ }^{2}$ S. K. Lam, J. B. Delos, R. L. Champion, and L. D. Doverspike, Phys. Rev. A 9, 1828 (1974).

${ }^{3}$ J. N. Bardsley, Proc. Phys. Soc. London 91, 300 (1967); J. Phys. B 1, 349 (1968); A. Herzenberg, Phys. Rev. 160, 80 (1967); C. J. Chapman and A. Herzenberg, J. Phys. B $\underline{5}, 790$ (1972).

4J. Mizuno and J. C. Y. Chen, Phys. Rev. 187, 167 (1969); Phys. Rev. A 4, 1500 (1971); J. C. Y. Chen, Advances in Radiation Chemistry, edited by M. Curton and J. L. Magee (Wiley, New York, 1968), Vol. 1, pp. 245ff; J. C. Y. Chen, Phys. Rev. 156, 12 (1967); J. C. Y. Chen and J. L. Peacher, ibid. 163, 103 (1967); 167, 30 (1968).

${ }^{5}$ See, for example, J. M. Blatt and V. F. Weisskopf, Theoretical Nuclear Physics (Wiley, New York, 1952).

6U. Fano, Phys. Rev. 124, 1866 (1961).

${ }^{7}$ Yu. N. Demkov and V. I. Osherov, Zh. Eksp. Theor. Fiz. 프,
1589 (1969) [Sov. Phys._JETP 26, 916 (1968)]; Yu. N. Demkov, Dok. Akad. Nauk. SSSR 166, 1076 (1966) [Sov. Phys.Dokl. 11, 138 (1966)].

${ }^{8}$ Local-complex-potential models involving electrostatic coupling between bound and free states allow $\Gamma$ to depend on the internuclear separation, but not on the internuclear velocity.

${ }^{9}$ R. L. Champion, L. D. Doverspike, and S. K. Lam, Phys. Rev. A 13, 617 (1976).

${ }^{10} \mathrm{~A}$ theory based on very different assumptions (the zerorange-potential model) has been used with considerable success to describe electron detachment. See J. P. Gauyacq, J. Phys. B 12, L387 (1979); 13, L501 (1979); 13, 4417 (1980).

${ }^{11}$ After many half-lives, an unstable state may decay nonexponentially; see, for example, R. G. Winter, Phys. Rev. 123, 1503 (1961). However, this fact cannot explain the observed isotope effects in negative-ion collisions.

${ }^{12}$ The discontinuous derivatives that necessarily occur in the 
static complex potential make an appearance in the work of Domcke and Cederbaum, and in this context our proof that the dynamical complex potential has continuous derivatives may have some interest. See W. Domcke, J. Phys. B 14, 4889 (1981); W. Domcke and L. S. Cederbaum, Phys. Rev. A 16, 1465 (1977); J. Phys. B 13, 2829 (1980); 14, 149 (1981).

${ }^{13}$ T. S. Wang, Ph.D. thesis, College of William and Mary, 1983; T. S. Wang and J. B. Delos, J. Chem. Phys. 79, 4306 (1983).

${ }^{14} E_{0}$ represents the largest penetration of the negative-ion curve into the continuum; the value taken here, about $5.5 \mathrm{eV}$, is at the high end of the normal range. $\beta$ is related to

$$
\frac{d^{2} \Delta}{d t^{2}}=\frac{d \Delta}{d R} \frac{d^{2} R}{d t^{2}}=\frac{d \Delta}{d R} \frac{\bar{F}}{\mu}
$$

at the turning point. If $d \Delta / d R$ and the averge radial force $\bar{F}$ are both a few electron volts per Bohr radius, and $\mu$ is around 2000 electron masses, then $10^{-5}$ is the appropriate order of magnitude for $\beta$.

${ }^{15}$ E. C. G. Stueckelberg, Helv. Phys. Acta $\underline{5}, 369$ (1932). 